\title{
INFLUENCIA DEL
}

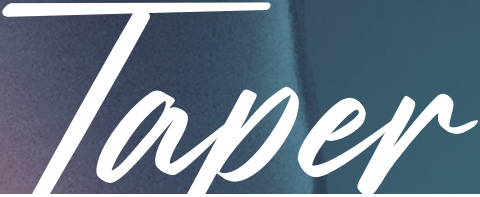

EN EL PORCENTAJE DE ÁREAS RELLENAS CON GUTAPERCHA, CEMENTO Y ESPACIOS VACÍOS EN CONDUCTOS OBTURADOS CON TÉCNICA HIIBRIDA DE TAGEER

INFLUENCE OF THE TAPER IN THE PERCENTAGE OF AREAS FILLED WITH GUTTA-PERCHA, CEMENT AND EMPTY SPACES IN SEALED ROOT CANALS WITH TAGGER HYBRID TECHNIQUE
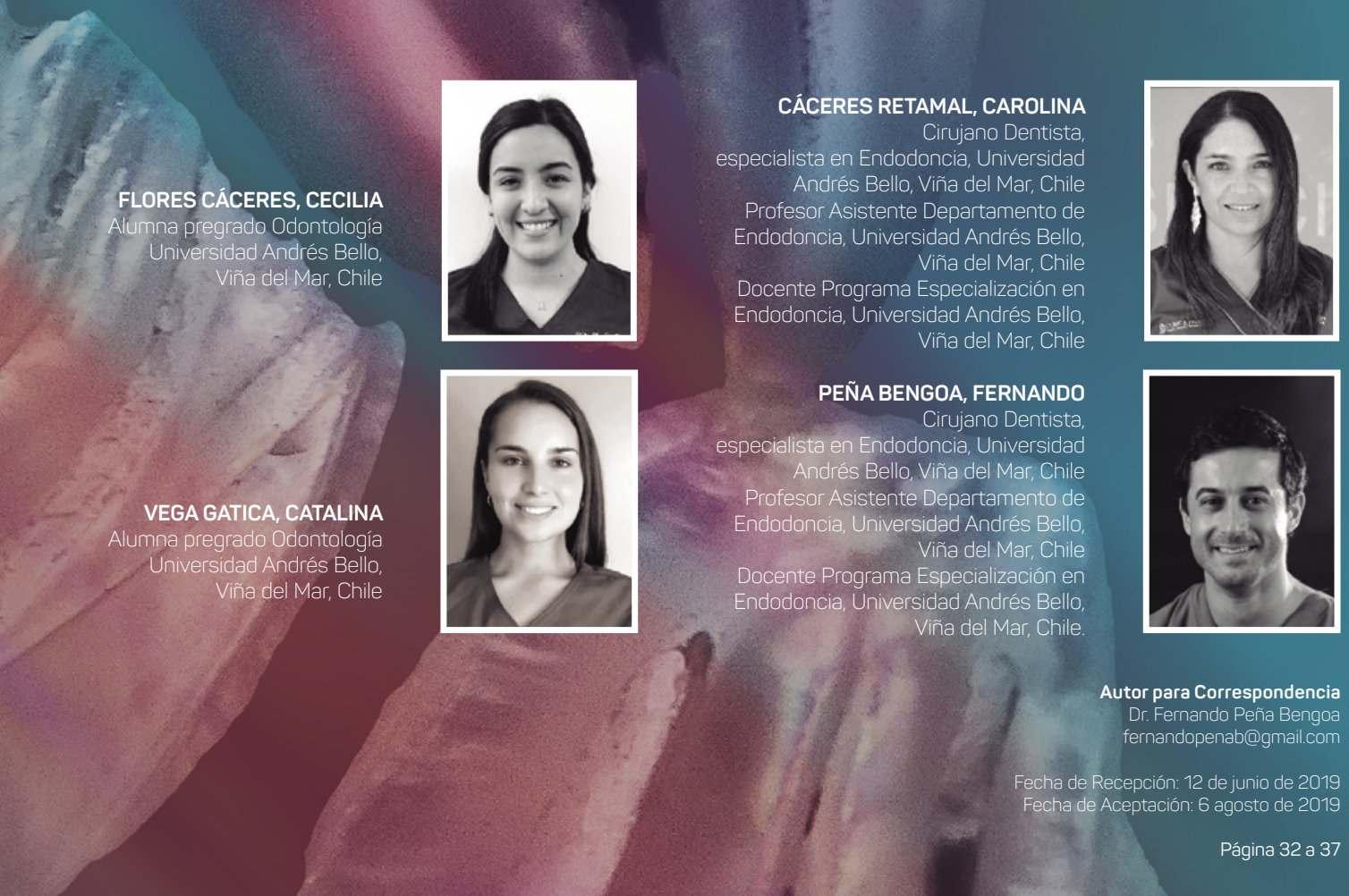

PEÑA BENGOA, FERNANDO

Cirujano Dentista,
Pencia Universidad especialista en Endodoncia, Universidad Profesor Asistente Departamento de Endodoncia, Universidad Andrés Bello, Endodoncia, Universidad Andres Bello,
Viña del Mar, Chile Docente Programa Especialización en Endodoncia, Universidad Andrés Bello Viña del Mar, Chile

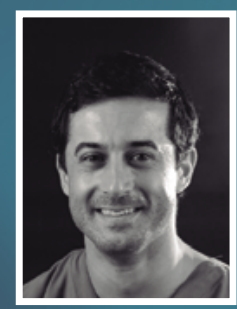

Autor para Correspondencia

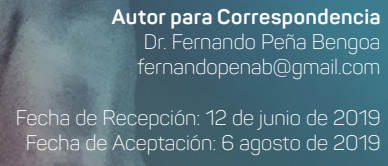

Página 32 a 37

\section{RESUMEN}

El objetivo del presente estudio experimentalin vitro, fue evaluar el porcentaje de áreas rellenas con gutapercha, cemento y espacios vacíos en la obturación radicular utilizàndo la técnica híbridá de Tagger comparando preparaciones con distintos taper.51premolares fueron divididos aleatoriamente en 3 grupos ( $n=17$ ), instrumentados con sistemas mecanizados con taper 4,6 y $8 \%$ y obturados con la técnica híbrida de Tagger. Los dientes fueron seccionados transversalmente a 2, 4 y 6 mm delápice, observados bajo microscopio óptico y medidos a través de un software de análisis de imagen. Los datos se analizaron mediante las pruebas Shapiro-Wilks, ANOVA, Kruskal-Wallis y Friedman. No se en-

contraron diferencias significativas al comparar el taper de conformación del conducto y los parámetros analizados con la técnica híbrida de Tagger. En conclusión, las conformaciones con un taper igual o mayor a $4 \%$ son adecuadas para obturar los conductos radiculares con la técnica híbrida de Tagger.

PALABRAS CLAVE: Taper, Técnica híbrida de Tagger, Gutapercha, Cemento sellador, espacios vacíos 


\section{ABSTRACT}

The aim of this experimental in vitro study, was to evaluate the percentage of areas filled with gutta-percha, sealer, and the voids areas on the root filling using hybrid Tagger technique comparing preparations with different tapers. 51 premolars were divided randomly in 3 groups ( $n=17)$, instrumented with mechanized systems with 4, 6, and 8\% taper, and filled with hybrid Tagger technique. The teeth were crosscut in 2, 4, and $6 \mathrm{~mm}$ from the apex, observed under an optical microscope, and measured through an image analysis software.

Data was analyzed with Shapiro-Wilks, ANOVA, Kruskal-Wallis and Friedman tests. No significant differences were found comparing the taper of the preparation and the analyzed criteria with hybrid Tagger technique. In conclusion, conformations with taper equal to or higher than $4 \%$ are suitable for root filling with hybrid Tagger technique.

KEY WORDS: Taper, Hybrid Tagger Technique, Gutta-percha, Sealer, Voids.

IMPLICANCIAS CLÍNICAS: El uso de instrumental con taper aumentado contribuye a una adecuada obturación de los conductos radiculares evitando espacios en la masa obturadora.

\section{INTRODUCCIÓN}

Aproximadamente el 60\% de los fracasos endodónticos son causados por una obturación deficiente del conducto radicular por falta de sellado1.La obturación radicular apunta a sellar tridimensionalmente el sistema de conductos, permitiendo a la gutaperchaentrar en máximo contacto con las paredes, relegando al cemento sellador solo a ocupar espacios pequeños en los cuales la gutapercha no sea capaz de ingresar 2. El taper aumentado de los sistemas de preparación actual,permiten generar un desgaste conservador en la zona apical y una mayor conicidad cervical lo cual favorece la adaptación del cono de gutapercha a las paredes del conducto, contribuyendo a una obturación homogénea, con una mínima cantidad de cemento sellador y libre de espacios vacíos que puedan alterar el pronóstico del tratamiento3-4.Las técnicas de obturación termoplásticas tienen la capacidad de reproducir la superficie interna del conducto obteniendo mejores resultados en el sellado tridimensional, mejorando el pronóstico del tratamiento 5. En este grupo encontramos la técnica termomecánica híbrida de Tagger, la cual combina la simplicidad de la técnica de compactación en frío con las ventajas de las técnicas termoplásticas.La homogeneidad y adaptación de la gutapercha a las paredes del conducto podría estar condicionada por el tipo de taper, sin embargo, aún falta evidencia científica que relacione la obturación radicular con el taper mínimo de la preparación.

El propósito de este trabajo es evaluar si el taper de la preparación del conducto influye en la obturación radicular en referencia al porcentaje de áreas rellenas con gutapercha (PAG), porcentaje de áreas rellenas con cemento sellador (PAC) y porcentaje de espacios vacíos (PEV) en dientes obturados con la técnica híbrida de Tagger a 2, 4 y 6 mm del ápice.

\section{MATERIALES Y MÉTODOS}

La muestra de esteestudio experimental in vitro, fue constituida por primeros y segundos premolares superiores e inferiores unirradiculares ysin curvaturas acentuadas extraídos por indicación ortodóncica durante el primer semestre del año 2018 en diversas instituciones de la $\mathrm{V}$ región (Chile). Estos fueron conservados en suero fisiológico inmediatamente posterior a su extracción. La anatomía de los conductos radiculares fue verificada por la toma simultanea de un Cone Beam (Gendex, gx CB 500 powered by I-Cat), para determinar la anatomía interna de la muestra y así excluir aquellos que tuvieran conductos ovalados en su tercio medio y apical.Las muestras fueron desbridadas y desinfectadas con hipoclorito de sodio 2,25\%.Serealizó el acceso y la longitud de trabajo (LT) se obtuvo de forma visual.

Las muestras se dividieron aleatoriamenteen 3 grupos ( $n=17)$ siendo: Grupo A: Lima ProDesing Logic (Easy) 25/04, Grupo B: Lima ProDesing Logic (Easy) 25/06 y Grupo C: Lima Reciproc Blue R25 (VDW) 25/08. El accionamiento de los instrumentos se realizó con un motor X-Smart plus (Dentsply, Maillefer)según las indicaciones del fabricante. Durante toda la preparación seutilizaron10 ml de $\mathrm{NaOCl}$ al 2,25\% y la irrigación final se realizó con EDTA al 17\% (10 ml) yde $\mathrm{NaOCl} 2,25 \%$ (10ml) según el protocolo de 
Schäfer y cols. (2013)6.Para la obturación radicular se utilizaron conosde gutapercha coincidentes con el instrumento de termino utilizado en la preparacióny se utilizóTOPSEAL (Dentsply-Maillefer) como cemento sellador. Se utilizaron 3 conos accesorios ISO \#25, espaciados con un spreader $N^{\circ} 30$ graduado a LT - 3 mm y con un termo compactador mecánico número 40 (Gutta Condensor, Dentsply-Maillefer)graduado a LT - 5mm se realizó el proceso de obturación radicular.Los dientes obturados fueron montados en bloques de acrilico transparente en su porción coronaria y almacenados en una incubadora (Cultura, Ivoclar Vivadent) durante 14 días a $37^{\circ} \mathrm{C}$. Los dientes fueron seccionados con una cortadora de precisión (IsoMet 5000 Precision Cutter - Buehler) a 2, 4 y 6 mm del ápice dentario(Figura 1).

Los cortes se observaron bajo un microscopio óptico BX43 (Olympus), donde se obtuvo una imagen digital de la superficie del corte con una ampliación de 10X. El PAG, PAC y PEV de cada corte, fueron medidos en micrómetros cuadrados utilizando un software de análisis de imagen (Micrometrics SE Premium) previamente calibrado. Las medidas de las áreas obtenidas se convirtieron a porcentajes del total de la zona, para facilitar el análisis de las muestras. (Figura 2).

Los datos se analizaron mediante los test de ANOVA, Kruskal-Wallis y Friedman con el software estadístico Stata 11.2. con un nivel de significancia estadística del 95\% (p<0.05).

\section{RESULTADOS}

No se encontraron diferencias significativas en términos de PAG, PAC y PEV (gráfico 1,2,3)en los cortes a 2, 4 y 6 mm del ápice. Por otro lado, al comparar los cortes radiculares obtenidos a $6 \mathrm{~mm}$ del ápice se pudo observar que,solo aquellos preparados con un taper del 6\%, presentaron diferencias significativas en términos de PAG y PAC (gráfico 1 y 2), no así en términos de PEV (gráfico 3).

\section{DISCUSIÓN}

El taper utilizado para la preparación del conducto no influyó en el PAG, PAC y PEV obtenidos en la obturación con la técnica híbrida de Tagger, por lo tanto, la hipótesis nula fue aceptada. Los resultados obtenidos conciden con los hallazgos del estudio realizado por Schäfer y cols (2016)3que, pese a que compararon distintas técnicas de obturación, no encontraron influencia en la obturación en términos de PAG, PAC y PEV al comparar preparaciones con distintos taper3.

La inexistencia de diferencias en relación a PAG y PAC, se pueden asociar a la técnica de obturación empleada en este estudio. Esta técnica, al utilizar un instrumento que genera calor por fricción, permite plastificar y generar el flujo de gutapercha hacia apical y lateral7. A su vez, el uso de conos de gutapercha coincidentes con los instrumentos de taper aumentado, mejora la adaptación de la gutapercha a las paredes del conducto; aumenta el volu- men de gutapercha y su homogeneidad, y por lo tanto reduce la cantidad de cemento sellador8.

Los resultados obtenidos en relación al PEV, se contradicen con el estudio realizado por Zogheib y cols. (2012)9, quien reportó que el PEV fue estadísticamente mayor en los grupos preparados con instrumentos de menor taper en comparación a aquellos de mayor9. Esta diferencia en los resultados se puede asociar, como se mencionó anteriormente, a características propias de la técnica de obturación empleada.

Los resultados obtenidos en el porcentaje de áreas rellenas con GP y el porcentaje de áreas rellenas de cemento en el grupo de taper 06 a los 6 mm, se contradicen con un estudio realizado por Schäfer y cols. (2016)3, donde no se obtuvieron diferencias significativas en término de PAG y PAC. En el año 2013, el mismo autor, comparópreparaciones con distintos sistemas y obturados con técnica de cono único, no encontrando diferencias significativas en términos de PAG y PAC. Por lo tanto, las diferencias obtenidas en esta investigación podrían estar asociadas a factores relacionados con la preparación de las muestras y a la anatomía interna propia de la muestra utilizada.

Cabe destacar que si bien el taper no influyó en la obturación radicular, según Paraskevopoulou M. y Khabbaz M. (2016)10 a mayor taper de preparación mayor volumen y profundidad de penetración de los irrigantes lo que se traduce en una mayor reducción bacteriana. De la misma forma, Buchanan L. (2002)11, describe que una preparación del sistema de conductos radiculares con taper aumentado, permite crear un espacio suficiente para optimizar el flujo de los irrigantes y la compactación del material de obturación. Por lo tanto, el éxito del tratamiento de endodoncia si puede ser influenciado por el taper de la preparación

\section{CONCLUSIÓN}

Tomando en consideración los resultados de ese estudio, todos los sistemascon taper igual o mayor a 4\% serían adecuados para obturar el sistema de conductos radiculares utilizando la técnica híbrida de Tagger. 


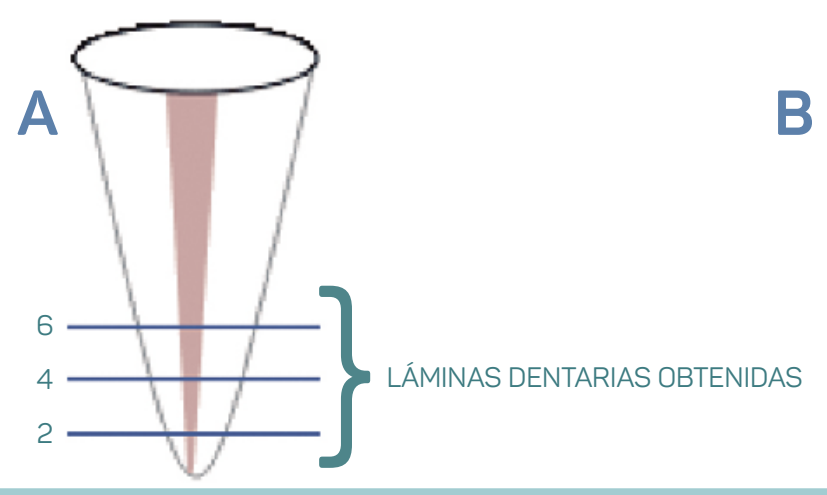

B

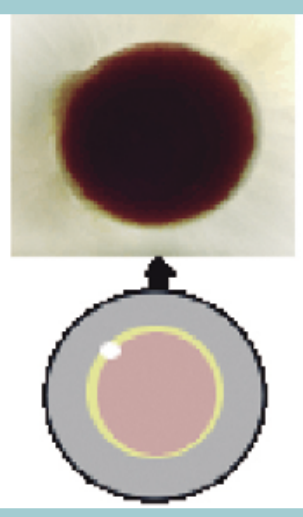

Figura 1.

Cortes radiculares. Representación gráfica de

os cortes radiculares (Imagen A). Corte radicular

a los $2 \mathrm{~mm}$ del ápice dentario (Imagen B).

Figura 2.

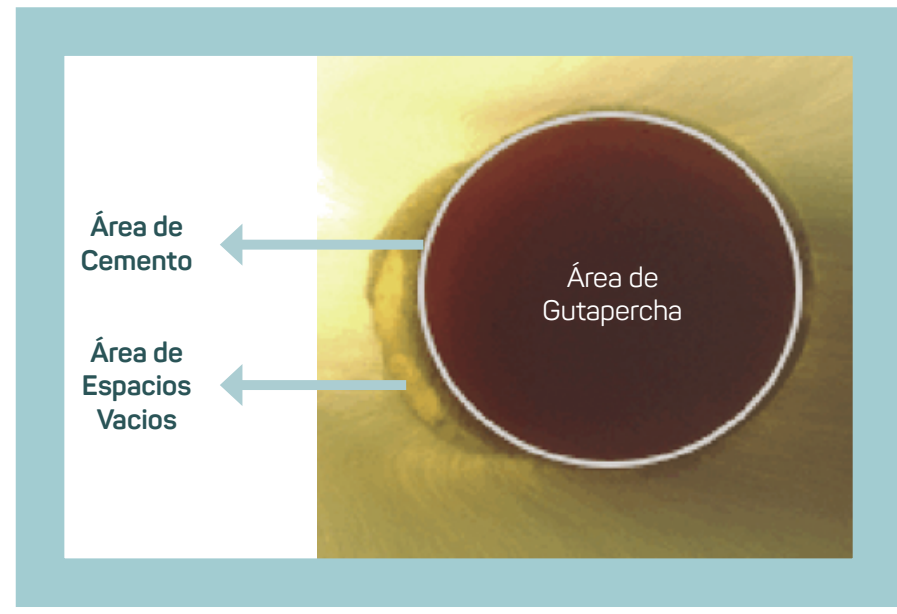

Corte radicular al microscopio óptico. Corte

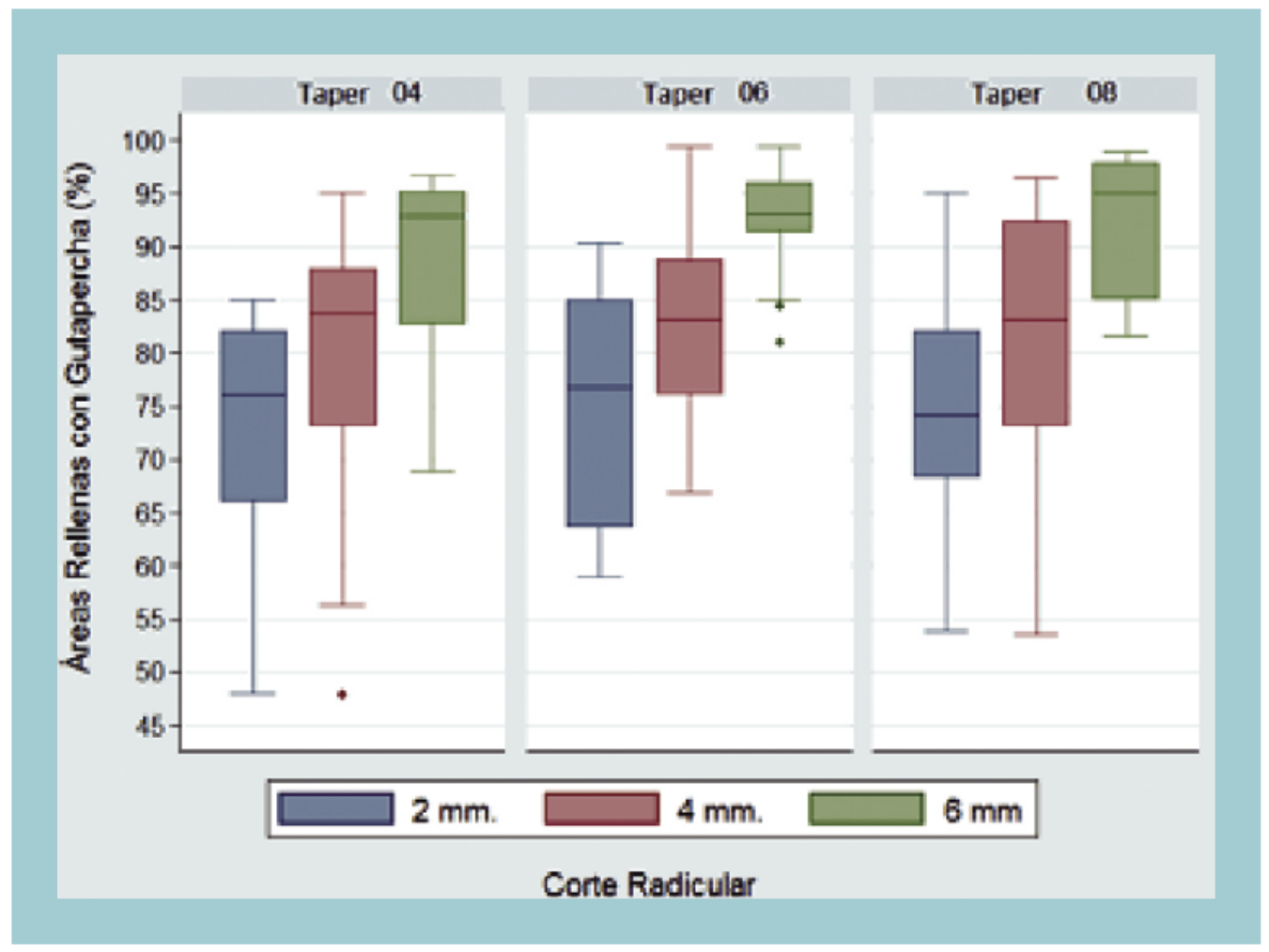

Gráfico 1.

Porcentaje de las áreas rellenas con gutapercha por taper y por corte radicular. 


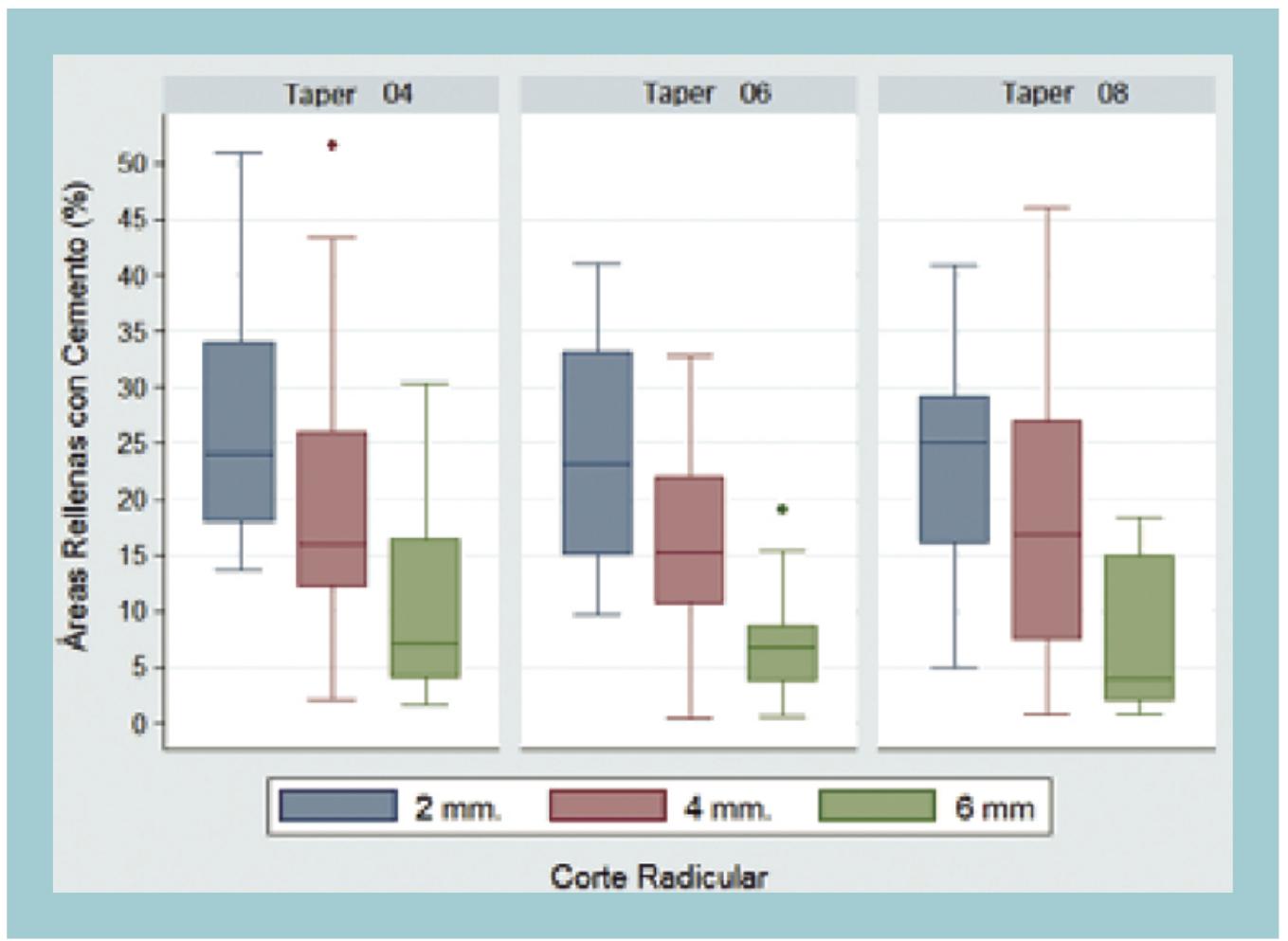

Gráfico 2

Porcentaje de las áreas rellenas con

cemento por taper y por corte radicular.

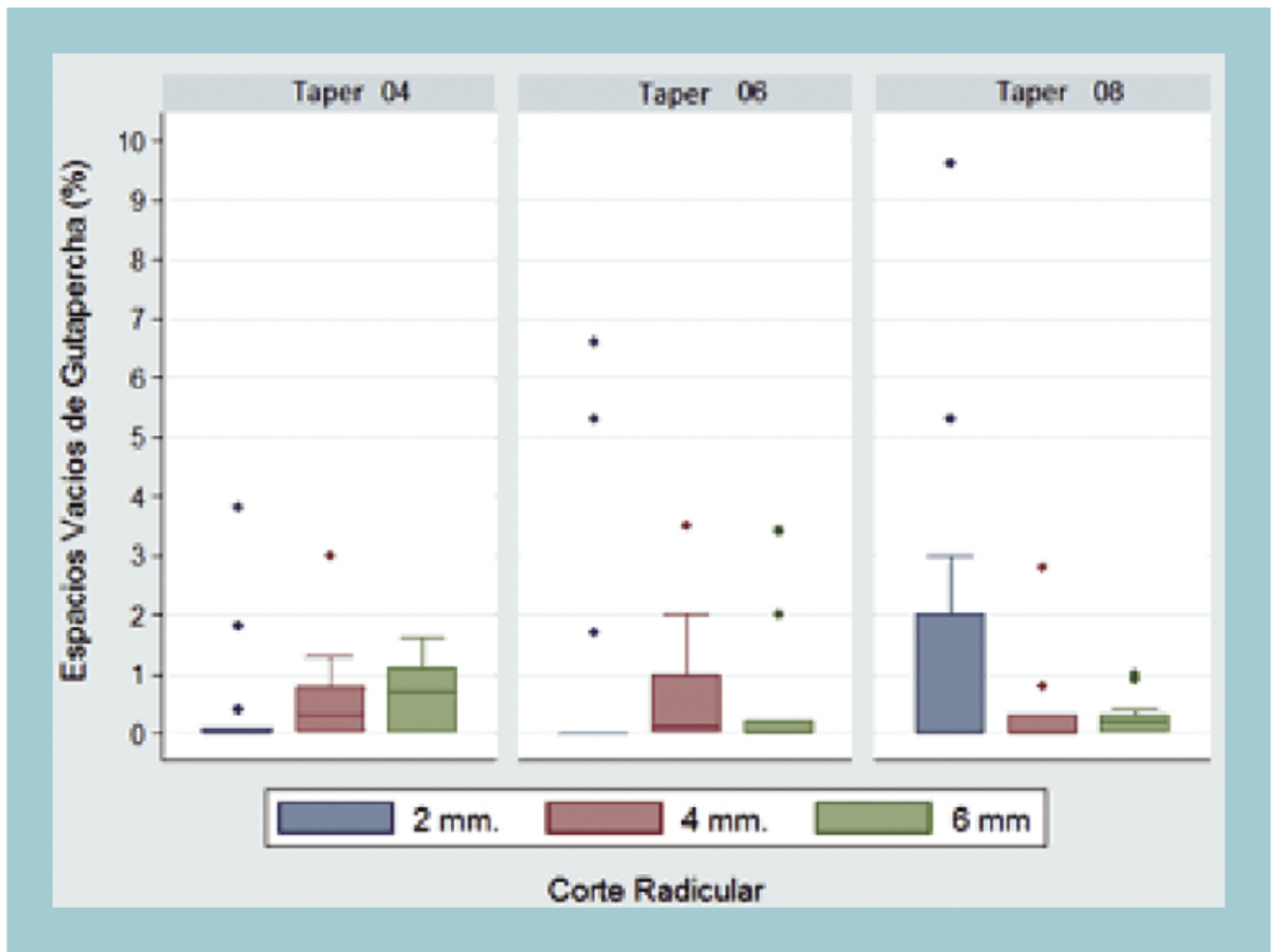

Gráfico 3

Porcentaje de espacios vacíos portaper y por corte radicular. 


\section{REFERENCIAS BIBLIOGRÁFICAS}

1. Monardes H, Abarca J, Castro P. Microfiltración apical de dos cementos selladores: un estudio in vitro. Int. J. Odontostomat. 2014, Dic;8(3): 393-8.

2. Libonati A, Montemurro E, Nardi R, Campanella V. Percentage of gutta-percha-filled areas in canals obturated by 3 different techniques with and without the use of endodontic sealer. JOE. 2017, Dic;44(3)506-

3. Schafer E, Schrenker C, Zupanc J, Burklein S. Percentage of gutta-percha filled areas in canals obturated with Cross-linked gutta-percha Corecarrier systems, Single-cone and Lateral compaction technique. JOE. 2016, Feb;42(2): 294-8.

4. Waplington M, McRobert A. Shaping the root canal sustem. Br. Dent. J. 2014, Mar;216(6):293-7.

5. Moradas M, Alvarez B. Do thermoplastic materials improve the obturation of the root canal? Bibliographic review of the different techniques available in the market. JDHODT. 2018, Jan;9(1): 00323

6. Schafer E, Koster M, Burklein S. Percentage of gutta-percha-filled areas in canals instrumented with Nickel-Titanium systems and obturated with matching single cones. JOE. 2013, Jul; 39(7)924-8.

7. Carrotte P. Endodontics: Part 8. Filling the root canal system. Br Dent J. 2004 Dec 11; 197(11): 667- 72

8. Bidar M, Sadeghi G, Gharechahi M, Mortazavi M, Forghani M. In vitro comparison of apical leakage in root canals obturated with 0.04 and 0.02 tapered guttapercha. Iran Endod J. 2010; Jun;5(3):97-100

9. Zogheib C, Naaman A, Medioni E, Arbab-shirani R. Influence of apical taper on the quality of thermoplasticized root fillings assessed by micro-computed tomography. Clin. Oral. Invest. 2012, Oct;16(5):1493-8

10. Paraskevopoulou M, Khabbaz M. Influence of taper of root canal shape on the intracanal bacterial reduction. Open Dent J. 2016; Oct;10: 568-74

11. Buchanan L. Innovations in endodontics instruments and techniques: how they simplify treatment. Dent Today. 2002. Dec, 21(12):52-4,56-61. 